\section{Estrategias y determinantes para recuperar participantes tras 12 años: cohorte CHICAMOCHA, Bucaramanga, Colombia}

\author{
Strategies and determinants for recovering \\ participants after 12 years: CHICAMOCHA \\ cohort, Bucaramanga, Colombia
}

\author{
Estratégias e determinantes para recuperar \\ participantes após 12 anos: coorte \\ CHICAMOCHA, Bucaramanga, Colômbia
}

Skarlet Marcell Vásquez 1

Victor Mauricio Herrera 1

Juan Carlos Villar 1

doi: 10.1590/0102-311X00059218

\section{Resumen}

Este artículo describe los resultados de un protocolo de recuperación de participantes (PRP) en la cohorte CHICAMOCHA, tras 12 años desde el último seguimiento, e identificar factores asociados al re-contacto. En un estudio de cohorte prospectiva de 1.644 participantes en Bucaramanga, Colombia (edad media 36 años; desviación estándar $=8.5$ años; 63\% hombres), se implementó el PRP que incluyó 4 estrategias secuenciales: (1) contacto telefónico; (2) envío de correspondencia; (3) visita al domicilio; $y$ (4) publicación de avisos de prensa. Luego de los pasos 1-2, se buscó refrescar la información de contacto en bases de datos gubernamentales para reaplicar paralelamente la secuencia 1-2 en no contactados. Se calcularon tasas de contacto crudas y ponderadas por estrategia del PRP y el rendimiento global. Se estimó la asociación entre las características de los participantes en línea de base y su estado después del PRP mediante regresión logística múltiple. Se contactaron $1.258(76,4 \%)$ participantes; el $65 \%(n=825)$ por contacto telefónico. Las tasas de contacto ponderadas fueron: $41 \%$ contacto telefónico, 14,6\% correspondencia y $31 \%$ visitas. El contacto por avisos de prensa fue virtualmente nulo. Una edad $>36$ años $(O R=1,48)$; estrato socioeconómico bajo $(O R=1,42)$ y ser propietario de vivienda en la línea de base $(O R=2,05)$ se asociaron al re-contacto. Consistente con otros reportes, el contacto telefónico es la estrategia de re-contacto con mayor rendimiento en estudios longitudinales. Los individuos con características que aumentan la movilidad geográfica podrían requerir de periodos de seguimiento más estrechos y estrategias de contacto adicionales.

Estudios de Seguimientos; Selección de Pacientes; Cooperaccion del Paciente; Negativa al Tratamiento

\author{
Correspondencia \\ S. M. Vásquez \\ Grupo de Cardiología Preventiva, Facultad de Ciencias \\ de la Salud, Universidad Autónoma de Bucaramanga. \\ Calle 157 \# 19-55, Floridablanca / Santander -681003, \\ Colombia \\ svasquez196@unab.edu.co \\ 1 Facultad de Ciencias de la Salud, Universidad Autónoma de \\ Bucaramanga, Bucaramanga, Colombia.
}




\section{Introducción}

Los estudios longitudinales con periodos de seguimiento a largo plazo han permitido responder importantes preguntas sobre la distribución de las enfermedades o sus determinantes en diferentes poblaciones. Las pérdidas durante el seguimiento pueden introducir sesgo de selección, cuando la muestra que es seguida difiere de la no seguida, con respecto a la distribución de predictores de los desenlaces de interés o variables confusoras. Por lo tanto, minimizar la proporción de observaciones censuradas resulta imprescindible para mantener la validez de las inferencias, lo que implica en primera instancia mantener el contacto con los participantes 1. La mayoría de los estudios longitudinales han sido realizados en países con altos ingresos, en los que el avanzado desarrollo e integración de los sistemas de información ha facilitado el contacto y seguimiento prospectivo de participantes, incluyendo la recolección de datos a partir de fuentes secundarias. En contraste a lo que ocurre en países de menores ingresos, como es el caso de Colombia 2,3, la investigación cuenta con una mayor y más sostenida financiación 4,5, lo que permite un contacto más frecuente con los participantes de los estudios y menores intervalos entre evaluaciones, favoreciendo su adherencia al seguimiento. Adicionalmente, particularidades del contexto social colombiano como lo es una marcada migración 6 (tanto interna como externa), aunado a recientes cambios regulatorios, relacionados con el manejo de datos personales 7 imponen retos aún mayores a los investigadores para mantener el contacto y minimizar las pérdidas durante el seguimiento.

El estudio Cardiovascular Health Investigation and Collaboration to Assess the Markers and Outcomes of Chagas Disease (CHICAMOCHA) es una cohorte de donantes de sangre, residentes en Bucaramanga, Colombia. Su objetivo principal es anidar diferentes estudios de historia natural o tratamiento en los participantes inicialmente libres de enfermedad cardiovascular. El reclutamiento en línea de base se inició en el año 2000 y fue terminado en el año 2003. En el año 2013, se logró financiación para ejecutar un nuevo protocolo de contacto y seguimiento en un subgrupo de la cohorte (participantes seronegativos Trypanosoma cruzi y los otros agentes evaluados en los bancos de sangre). El proyecto buscaba informar la incidencia y factores de riesgo, asociados a la hipertensión arterial en la cohorte CHICAMOCHA, tras diez años de seguimiento. Este objetivo requería como prerrequisito el planteamiento y ejecución de un protocolo de recuperación de participantes (PRP). Se estableció como meta recuperar 70\%-75\% de los participantes (aproximadamente 1.200 personas para identificar unos 300 casos con hipertensión), con lo que además esta cohorte tuviese continuidad y viabilidad como activo institucional y social.

El propósito principal de este estudio es describir las estrategias, procedimientos y resultados obtenidos con el PRP del estudio CHICAMOCHA, tras 12 años después del último contacto. El objetivo secundario fue determinar los factores que se asociaron al re-contacto de los participantes.

\section{Métodos}

\section{Población de estudio}

CHICAMOCHA es una cohorte de 2.133 donantes de sangre con edad $\geq 18$ años: 489 con pruebas serológicas de tamización positiva para T. cruzi y 1.644 con serología negativa a todos los agentes evaluados antes de la donación. Dentro de la cohorte se ha adelantado un estudio diagnóstico (por comparación de participantes seropositivos con una muestra aleatoria de seronegativos) 8 y se adelantan dos ensayos clínicos en el grupo de participantes con serología positiva a T. cruzi (Villar JC, Oct/2010, comunicación personal) 9,10. En tanto, la evolución del grupo con serología negativa, sin nuevas visitas de seguimiento, es una fuente potencial de importante información de tipo diagnóstico y pronóstico aún sin aprovechar.

El presente estudio se enmarca en los participantes con pruebas serológicas negativas, considerados como adultos clínicamente sanos, libres de enfermedad cardiovascular por evaluación clínica en la línea de base. Los detalles acerca del proceso de ensamblaje de la cohorte, las características de los participantes y las mediciones hechas se han publicado previamente 11. Brevemente, se partió de una muestra consecutiva de todos los donantes positivos a las pruebas para T. cruzi (y negativas para las 
demás pruebas serológicas) en los bancos de Bucaramanga. El grupo seronegativo fue seleccionado a partir de una base de datos de donantes de bancos de sangre, con pruebas de tamización serológica negativas en ese mismo periodo. A partir de allí, se obtuvo una muestra aleatoria de 4 donantes negativos por cada seropositivo, en el mismo mes de donación, grupo de edad y sexo de cada seropositivo; posteriormente, se reclutaron los 1.644 participantes que constituyen la población de este estudio.

\section{Antecedentes de seguimiento en la cohorte}

Aproximadamente 3 años después de la línea de base se adelantó un primer seguimiento. Buscando contacto telefónico, seguido por envío de cartas al domicilio y visitas al vecindario, se logró la asistencia de $1.230(74,8 \%)$ participantes. Tres años después, se intentó contacto con una muestra aleatoria de personas seronegativas $(\mathrm{n}=200)$, que servían como grupo de comparación en el estudio diagnóstico de seropositivos, contando con 171 (85,5\%) asistentes. Las limitaciones presupuestarias impidieron tener luego nuevos seguimientos con la totalidad del grupo.

\section{Protocolo de recuperación de participantes}

Basados en las experiencias previas de contacto y en el marco de los recursos disponibles, se implementó el PRP, basado en la aplicación de 4 estrategias, así: (1) contacto telefónico; (2) envío de correspondencia al domicilio y correo electrónico; (3) visita al domicilio y vecindario; y (4) publicación de avisos de prensa. Cada una de estas estrategias fue registrada sistemáticamente en un aplicativo electrónico de captura de información, que clasificaba por módulos los participantes elegibles para cada etapa. Los procedimientos usados para la aplicación de las estrategias son descritos a continuación.

\section{Contacto telefónico}

Se realizaron hasta 5 intentos de contacto en cada número telefónico registrado, en diferentes horarios, incluyendo días entre semana y fines de semana. La Figura 1 presenta el algoritmo usado para estos procedimientos.

Cada llamada se registró con el nombre del operador y sus observaciones respectivas. En cada llamada respondida por una persona que no conocía al participante se verificó si la dirección de domicilio registrada como residencia del participante correspondía a ese número telefónico, con el objetivo de identificar direcciones hábiles (nuevo sitio de residencia o trabajo) para continuar con los procesos de contacto.

Si durante el proceso de llamadas se lograba contactar directamente con el participante, el operador debía actualizar su dirección y número telefónico de la residencia, número celular, dirección y teléfono del trabajo, además de registrar todos los números telefónicos de personas de contacto posible con el participante y su parentesco. Después de actualizada, la información no validada era eliminada.

Cuando se lograba un contacto indirecto (con un familiar o conocido que pudiera dar referencias del participante), el operador indicaba el motivo de la llamada y dejaba los números de contacto con la oficina del proyecto. Si pasada una semana no se retornaba la llamada, se reintentaba contacto con el mismo número hasta 3 veces. Agotada esta instancia, el participante era etiquetado como no contactado, y trasladado automáticamente al módulo de envío de correspondencia al domicilio.

\section{Envío de correspondencia al domicilio}

Se envió carta por correo certificado a los participantes no contactados telefónicamente, cuyas direcciones de domicilio en línea de base no se encontraron relacionadas con los números telefónicos disponibles. El contenido de la carta recordaba al destinatario su carácter de participante voluntario y los objetivos del proyecto, invitándolo a ponerse en contacto con el proyecto. En el grupo de participantes que registró un correo electrónico en línea de base (2\%) se enviaron mensajes, además de la carta. Se esperó respuesta del participante a la carta hasta un mes después del envío. El grupo que 
Figura 1

Algoritmo de los procedimientos de contacto telefónico.

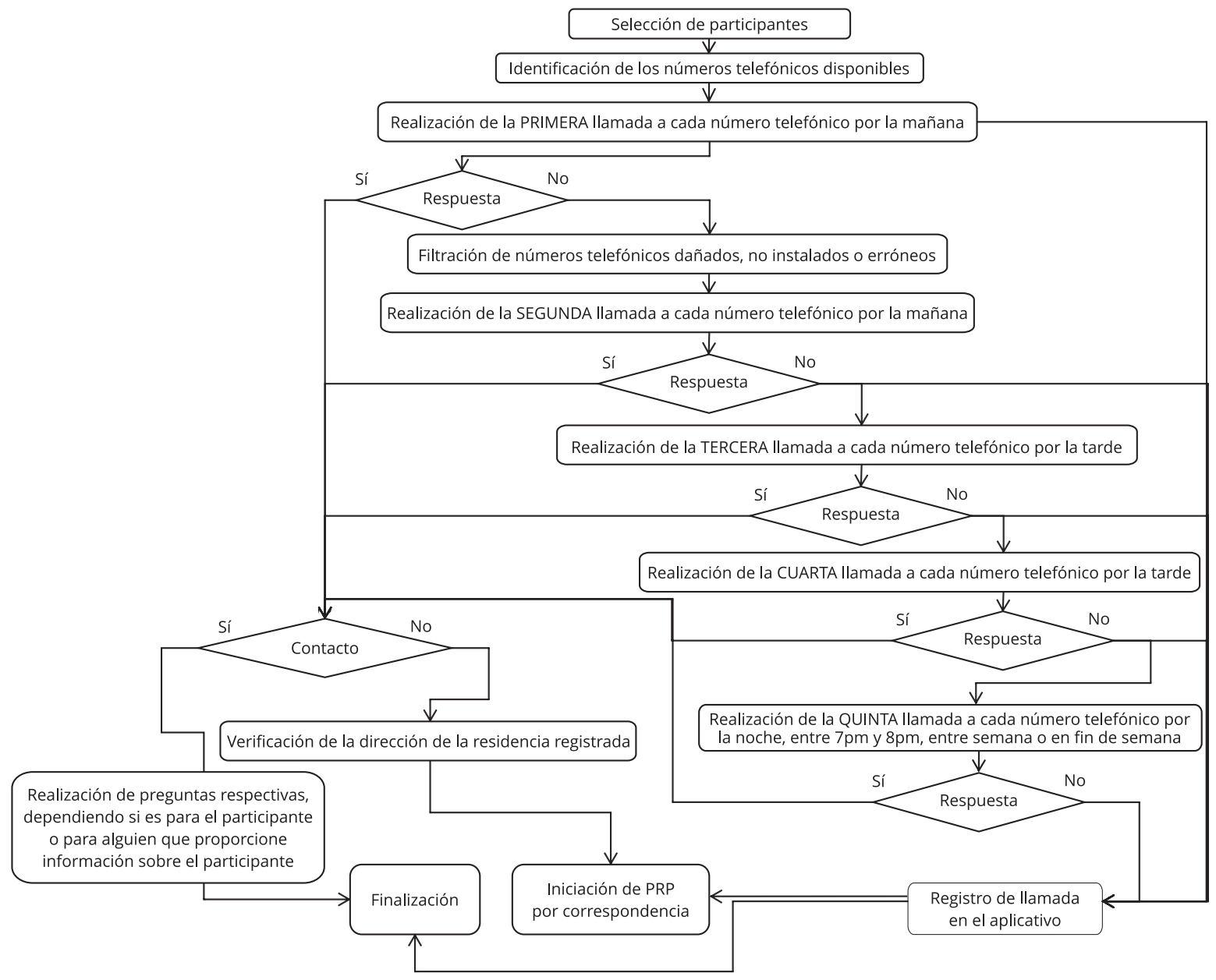

PRP: protocolo de recuperación de participantes.

no logró ser contactado a través de esta estrategia fue registrado como no contactado y trasladado al módulo siguiente.

\section{Visitas al domicilio y vecindario}

Para optimizar los recursos financieros, de personal y el tiempo de ejecución; todas las direcciones de los participantes que permanecían sin contacto fueron incorporadas a un sistema de georreferenciación, que generaba ubicaciones dentro del mapa de Bucaramanga y su área metropolitana (Girón, Floridablanca, Piedecuesta y Lebrija). Cada municipio fue dividido por zonas para identificar residencias cercanas; y conjuntamente cada residencia fue marcada con un radio de 200 metros para seleccionar grupos de viviendas que pudieran ser incluidos en la misma jornada de visitas por el mismo personal. Se establecieron tres cuadrillas integradas cada una por dos personas del proyecto, previamente entrenadas, para ejecutar sistemáticamente el siguiente proceso: (1) visitar la dirección de domicilio registrada para cada participante en línea de base. Si el participante no era contactado, o no se obtenían datos de ubicación por referencia, se visitaba el edificio siguiente, primero en el lado 
derecho del domicilio, luego del lado izquierdo, de no conseguirse contato; (2) de no lograrse contacto efectivo, se visitaba la tienda, negocio o micromercado más cercano al domicilio; y (3) finalmente, si en las visitas anteriores no se obtenían resultados, se preguntaba por el vecino de mayor antigüedad en el barrio para visitarlo.

En estos pasos, el personal del estudio realizaba entrevistas a los residentes de los edificios visitados, buscando obtener algún dato que permitiera contactar al participante. En el caso de conseguir por referencia nuevos datos telefónicos del participante, se reiniciaba el PRP desde el contacto telefónico.

\section{Publicación de avisos de prensa}

Al finalizar los pasos previos, se publicaron dos avisos en la página principal de los dos periódicos de mayor cobertura en Bucaramanga, buscando que los participantes aún no contactados se comunicaran con la oficina del proyecto. Se publicaron 2 avisos de prensa con un intervalo de 15 días, esperando aumentar su visualización por los participantes sin contacto.

\section{Ejecución del protocolo de recuperación de participantes}

\section{- Protocolo de recuperación de participantes 1}

El protocolo de contacto inicial incluyó el uso secuencial de las estrategias: contacto telefónico y envío de correspondencia al domicilio. Una vez se culminó la ejecución de estos pasos en todos los participantes de la cohorte, se buscó actualizar la información de contacto a través de las Entidades Administradoras de Planes de Beneficios (EAPB), por consulta a la base de datos única de afiliados al sistema general de seguridad social en salud (BDUA), para realimentar el proceso con un nuevo PRP (que se denominó PRP 2), y que se desarrolló en paralelo a la estrategia de visitas al domicilio y vecindario al primer grupo de PRP. Una vez culminadas las visitas y el PRP 2, se ejecutó la estrategia de aviso de prensa para abordar a los participantes no contactados.

\section{- Protocolo de recuperación de participantes 2}

Inicialmente, se hicieron consultas por número de identificación de los participantes no contactados previamente usando la BDUA. Hasta el año 2015, la BDUA estaba disponible en la página web del Fondo de Solidaridad y Garantía (FOSYGA). De acuerdo a los datos obtenidos, se registró el estado de afiliación de cada participante, la entidad a la que se encontraba afiliado, tipo de régimen y lugar de afiliación. Posteriormente, el proyecto solicitó autorización del Ministerio de Salud y Protección Social para establecer comunicación con las EAPB para la obtención de datos personales actualizados de los participantes, con propósito de esta investigación. Con este aval, se remitió desde la oficina del proyecto a cada entidad la lista de los participantes afiliados, copia del consentimiento informado del reclutamiento inicial del participante y solicitud de información de contacto de sus afiliados. En el caso de conseguir información actualizada de teléfono o domicilio de los participantes, el PRP 2 incluía el desarrollo de las estrategias de contacto en el mismo orden en que se ejecutaron en el PRP 1.

\section{Análisis estadístico}

Para informar el rendimiento de los PRP se reportaron las tasas de contacto de acuerdo con las definiciones estandarizadas de la Asociación Americana para la Pesquisa de la Opinión Pública 12. Para las estrategias del PRP 1 se estimaron las tasas de contato (CON1); se incluyeron en el numerador todos los participantes con los que se estableció contacto directo o indirecto, lo que permitió establecer su estado vital (en el caso de fallecidos la familia reportó la causa de muerte), con quienes se completó parcial o completamente la entrevista sin importar su ubicación geográfica. En el denominador se consideraron como elegibles todos los participantes de la cohorte que no habían sido contactados hasta el momento previo a iniciar una estrategia específica.

Para las estrategias del PRP 2 se estimaron las tasas CON3; en cuyo denominador sólo se consideraron como elegibles los participantes con quienes se logró actualización de datos, a través de la 
BDUA y las EAPB, o en el caso de la estrategia de visitas al domicilio y vecindario, sólo se consideraron en el denominador los participantes con quienes se pudo efectuar la visita.

Con el objetivo de considerar conjuntamente las dos PRP, en la estimación final de las tasas de contacto se calcularon tasas ponderadas, empleando como factor de ponderación para cada protocolo el cociente entre el número de participantes elegibles para su aplicación y el total de participantes abordados por las dos PRP, de tal manera que la sumatoria de factores fuese igual a uno.

Con el objetivo de determinar los factores asociados al re-contacto, se consideraron como variables potencialmente asociadas el género; la edad (dicotomizada de acuerdo a su distribución en $\leq 36$ años ó > 36 años para el análisis de regresión logística); el estado civil (se definió como casado a toda persona que viviera con pareja, independiente del estado legal de la unión); el estrato socioeconómico de la vivienda (definido de acuerdo a la clasificación colombiana en una escala de 1 a 6; siendo 1, 2 y 3 los estratos bajos y 4, 5 y 6 los estratos medio-altos); ser propietario de vivienda; el nivel educativo (categorizado como primaria, secundaria, técnico, universitario o posgrado); la percepción del participante del riesgo de enfermedad general, en comparación a la población general (determinada en una escala de 1 a 5, siendo 1 percepción de riesgo definitivamente menor y 5 percepción de riesgo definitivamente más alto); la asistencia y realización de chequeos médicos o de laboratorio de forma preventiva; la presencia de antecedentes médicos familiares y la presencia de comorbilidades en el participante. En un primer paso, se compararon estas características de los participantes en línea de base (medias y desviación estándar, o conteos y porcentajes, de acuerdo a las variables) y su situación al finalizar el protocolo. Se probaron hipótesis de no diferencias entre los grupos con la prueba t de student o de Mann-Whitney para variables continúas (según su distribución) o $\chi^{2}$ o test exacto de Fisher (según los eventos observados) para variables categóricas. En un segundo paso, se usó regresión logística múltiple para identificar asociaciones independientes entre características de línea de base seleccionadas ( $\mathrm{p}<0,20$ en el análisis bivariado) y el re-contacto, con nivel de significancia $\mathrm{p}<0.05$. La información fue analizada en el paquete estadístico Stata 12.1 (https://www.stata.com).

\section{Resultados}

La ejecución de los PRP en los 1.644 participantes se inició en mayo de 2013 y culminó en diciembre del año 2015. Luego de una mediana de seguimiento de 12,4 (rango intercuartílico 10-16) años, se contactó a $1.258(76,5 \%)$ participantes. Esto incluye 27 fallecimientos $(70,4 \%$ por causas no cardiovasculares) y 63 participantes residentes fuera de Bucaramanga. La Figura 2 muestra el flujo de ejecución de los PRP y las estrategias de contacto.

La estrategia de llamadas telefónicas en el PRP 1 fue desarrollada en la totalidad de los participantes; el 50\% de los cuales tenía registro de 3 (rango intercuartílico 2-4) números telefónicos y menos del $10 \%$ tenían registro de número celular. Al finalizar el protocolo, se obtuvo una tasa de contacto para esta estrategia del 47,5\% (781/1.644).

A 863 (52,5\%) participantes con contacto inefectivo, tras esta primera estrategia del PRP 1, se les realizó envío de correspondencia al domicilio. En un subgrupo de 304 participantes $(35,2 \%)$ se consideró necesario realizar un segundo envío de correspondencia, debido a que el primer envío se ejecutó a inicios del mes de diciembre de 2014, disminuyendo la oportunidad de recepción de respuestas de los participantes, en el caso de que estos contactaran con los investigadores en periodo de vacaciones, lo que llevó a estimar una tasa de contacto ponderada para esta estrategia en el PRP 1 (factor de ponderación 0,33 único envío vs. 0,66 doble envío). La tasa de contacto ponderada para esta estrategia fue de $18,6 \%$ en el PRP 1.

Los 629 (38,3\%) participantes que permanecían sin contacto, después de la ejecución de estas dos estrategias y el inicio del PRP 2 paralelo, fueron candidatos para la estrategia de visitas al domicilio y vecindario. Se realizaron 550 visitas (79 participantes no fueron visitados por vivir en zonas de alto riesgo o fuera de Bucaramanga), logrando contacto en 27,1\% (149/550) participantes.

En el PRP 2 paralelo, se consultaron en la BDUA del FOSYGA 647 (39,4\%) participantes, generando solicitudes de datos de contacto para 24 EAPB. Luego de algunos meses, se obtuvo respuesta solo de 5 entidades, permitiendo actualizar datos telefónicos o de dirección de domicilio para 366 (56,6\%) participantes. Una vez actualizada la información, las tasas de contacto para las estrategias en 


\section{Figura 2}

Flujo de ejecución de las estrategias de los protocolos de recuperación de participantes (PRP 1 y 2).

PRP 1

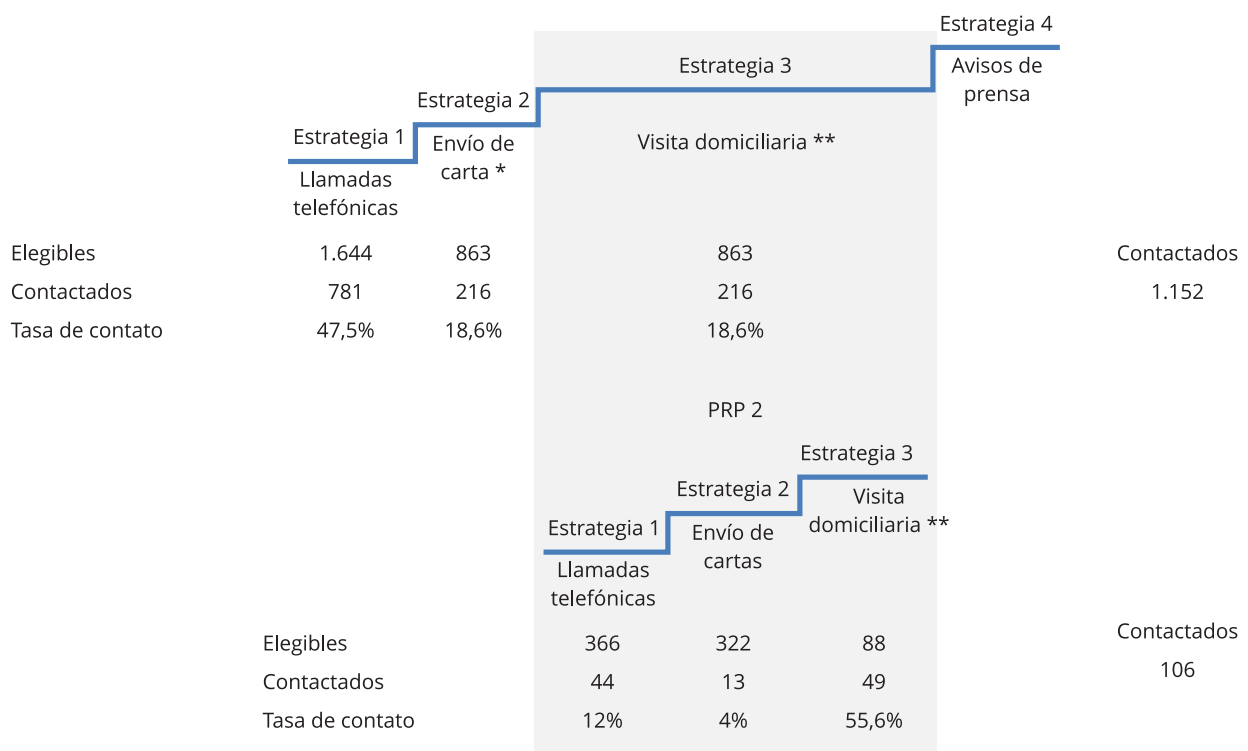

* Se estimó una tasa de contacto ponderada, teniendo en cuenta que 304 participantes tuvieron un doble envío de cartas (tasa de contacto en el subgrupo $=12,2 \%$ [37/304]).

** 79 participantes de los 647 sin contacto, no se visitaron por vivir en zonas de alto riesgo o se fueron de Bucaramanga.

el PRP 2 fueron: $12 \%$ contacto telefónico; 4\% envío de cartas al domicilio y 55,6\% visitas al domicilio y vecindario.

Finalmente, fueron objeto de los avisos de prensa $392(23,8 \%)$ participantes. Sólo $1(0,3 \%)$ estableció contacto con la oficina del proyecto posterior a estos anuncios, sin embargo, durante la ejecución de esta última estrategia también fueron localizados 5 participantes por referencia de sus pares durante la visita de seguimiento. La Tabla 1 resume las tasas de contacto crudas y ponderadas para cada estrategia, después de la ejecución de los PRP. La mayor proporción de participantes se logró contactar a través del contacto telefónico (tasa de contacto ponderada 41\%, 821 participantes contactados). Aunque el envío de correspondencia al domicilio permitió contactar a 229 participantes, la tasa de contacto ponderada fue superior para la estrategia de visitas al domicilio y vecindario ( $31 \% v s .14,6 \%$ ).

La Tabla 2 muestra las características de línea de base de acuerdo a la situación del participante al finalizar el protocolo. Se observó que los participantes recuperados tenían mayor edad promedio $(36,5$ vs. 33,6 años; $\mathrm{p}<0,001)$ y con mayor frecuencia residían en los estratos socioeconómicos 1-3 $(72,4$ vs 65,$8 ; \mathrm{p}=0,012)$, eran propietarios de vivienda $(52,4 \%$ vs. $35,2 \% ; \mathrm{p}<0,001)$ o habían asistido a chequeos médicos o de laboratorio preventivos $(38,4 \%$ vs. $31,1 \% ; \mathrm{p}=0,011)$. En el análisis multivariado, la edad mayor a 36 años, la media en línea de base $(\mathrm{OR}=1,48)$, residir en estratos 1 -3 $(\mathrm{OR}=1,42)$ y ser propietario de la vivienda $(\mathrm{OR}=2,05)$ se asociaron al contacto (Tabla 3$)$. 
Tabla 1

Tasas de contacto crudas y ponderadas de acuerdo a la estrategia, después de la implementación de los protocolos de recuperación de participantes (PRP) 1 y 2.

\begin{tabular}{|c|c|c|c|c|}
\hline \multirow[t]{2}{*}{ Estrategia de contacto } & \multicolumn{2}{|c|}{ Tasa de contacto } & \multirow{2}{*}{$\begin{array}{l}\text { Factor de ponderación } \\
\qquad N /(N 1+N 2)\end{array}$} & \multirow{2}{*}{$\begin{array}{c}\text { Tasa de contacto } \\
\text { ponderada } \\
\%\end{array}$} \\
\hline & $n / N$ & $\%$ & & \\
\hline Llamadas telefónicas & & & & 41,0 \\
\hline PRP 1 & $781 / 1.644$ & 47,5 & 0,82 & \\
\hline PRP 2 & $44 / 366$ & 12,0 & 0,18 & \\
\hline Envío de correspondencia & & & & 14,6 \\
\hline PRP 1 * & $216 / 863$ & 18,6 & 0,73 & \\
\hline PRP 2 & $13 / 322$ & 4,0 & 0,27 & \\
\hline Visita domiciliaria & & & & 31,0 \\
\hline PRP 1 & $149 / 541$ & 27,5 & 0,86 & \\
\hline PRP 2 & $49 / 88$ & 55,7 & 0,14 & \\
\hline Aviso de prensa & $6 / 392$ & 1,5 & & - \\
\hline Tasa de contacto global & $1.258 / 1.644$ & 76,5 & & \\
\hline
\end{tabular}

* Se estimó una tasa de contacto ponderada, teniendo en cuenta que 304 participantes tuvieron un doble envío de cartas (tasa de contacto en el subgrupo $=12,2 \%$ [37/304]).

Tabla 2

Características de la línea de base de acuerdo a la situación del participante al finalizar el protocolo de recuperación de participantes (PRP).

\begin{tabular}{|c|c|c|c|}
\hline Características en la visita de línea de base & $\begin{array}{l}\text { Contactados } \\
(n=1.258)\end{array}$ & $\begin{array}{l}\text { No contactados } \\
\quad(n=386)\end{array}$ & Valor de $p$ \\
\hline Género masculino [n (\%)] & $798(62,4)$ & $243(62,9)$ & 0,864 \\
\hline Edad media (DE) & $36,5(8,3)$ & $33,6(8,6)$ & 0,000 \\
\hline Estado civil casado [n (\%)] & $801(64,4)$ & $229(59,6)$ & 0,088 \\
\hline Estrato socioeconómico [n (\%)] & & & 0,012 \\
\hline $1-3$ & $911(72,4)$ & $254(65,8)$ & \\
\hline $4-6$ & $347(27,6)$ & $132(34,2)$ & \\
\hline Propietario de vivienda [n (\%)] & $659(52,4)$ & $136(35,2)$ & 0,000 \\
\hline Nivel educativo primaria o secundaria [n (\%)] & $770(62,1)$ & $233(60,3)$ & 0,529 \\
\hline $\begin{array}{l}\text { Percepción de riesgo alto de enfermedad general en comparación con población } \\
\text { general }[n(\%)]\end{array}$ & $564(44,8)$ & $161(41,7)$ & 0,280 \\
\hline Se realiza chequeos médicos o de laboratorio preventivos [n (\%)] & $451(38,4)$ & $115(31,1)$ & 0,011 \\
\hline \multicolumn{4}{|l|}{ Antecedentes familiares } \\
\hline Enfermedad cerebrovascular [n (\%)] & $41(3,5)$ & $20(5,4)$ & 0,093 \\
\hline Fallo cardíaco [n (\%)] & $73(6,1)$ & $22(5,9)$ & 0,877 \\
\hline Enfermedad coronari [n (\%)] & $62(4,9)$ & $16(4,2)$ & 0,527 \\
\hline Infarto cardíaco [n (\%)] & $29(2,3)$ & $11(2,9)$ & 0,544 \\
\hline \multicolumn{4}{|l|}{ Comorbilidades } \\
\hline Obesidad [n (\%)] & $583(46,3)$ & $168(43,5)$ & 0,331 \\
\hline Presión arterial sistólica > 140mmHg [n (\%)] & $46(3,6)$ & $12(3,1)$ & 0,610 \\
\hline Diabetes [n (\%)] & $23(1,8)$ & $7(1,8)$ & 0,985 \\
\hline
\end{tabular}

DE: desviación estándar. 
Tabla 3

Resultados del modelo para estimar la asociación entre las características de la línea de base preseleccionadas y la recuperación de contacto con participantes de la cohorte.

\begin{tabular}{lccc}
\hline Características en línea de base & OR & IC95\% & Valor de $\mathbf{p}$ \\
\hline Edad > 36 años & 1,48 & $1,14-1,91$ & 0,003 \\
Estado civil casado & 1,05 & $0,81-1,36$ & 0,708 \\
Estrato socioeconómico 1-3 & 1,42 & $1,08-1,84$ & 0,009 \\
Propietario de vivienda & 2,05 & $1,59-2,62$ & $<0,001$ \\
Chequeos médicos preventivos & 1,32 & $1,01-1,71$ & 0,037 \\
Antecedente familiar ACV & 0,68 & $0,38-1,21$ & 0,187 \\
\hline
\end{tabular}

ACV: accidente cerebrovascular; IC95\%: intervalo de 95\% de confianza; OR: odds ratio.

\section{Discusión}

Pese a los desafíos encontrados durante la ejecución de los PRP, la secuencia de estrategias usadas permitió recuperar el 76,5\% de los participantes del grupo seronegativo de la cohorte CHICAMOCHA. Se logró, después de más de una década, contacto -vía telefónica- con la mitad de los participantes (o el 65\% de los recuperados); estableciendo esta estrategia como la de mayor rendimiento del PRP e identificando el envío de correspondencia y visitas al domicilio como estrategias complementarias con aceptable rendimiento para el re-contacto.

Las experiencias previas de contacto en CHICAMOCHA nos permitieron plantear un PRP que incluía el uso de 4 estrategias conocidas. Sin embargo, la limitación en los recursos económicos disponibles nos llevó a reorientar el PRP inicial; ejecutando un PRP paralelo en el que la obtención de datos de contacto actualizados, a través de la BDUA y las EAPB, se convirtió en una herramienta clave para optimizar las estrategias de contacto en un subgrupo de participantes.

Otra modificación al PRP se generó durante el período de vacaciones de los investigadores, hacia el final del mes de diciembre de 2014; tiempo en el que se consideró que quienes habían sido objeto de la estrategia de envío de correspondencia, al inicio de ese mismo mes, pudieron establecer contacto con la oficina del estudio, sin obtener respuesta por la ausencia de los investigadores. Por este motivo, se determinó realizar un nuevo envío de correspondencia a inicios del año 2015 en un subgrupo de 304 participantes. Se estimó una mayor posibilidad de contacto en los participantes con doble envío de correspondencia, lo que llevó a que la tasa de contacto para esta estrategia en el PRP 1 se ponderará, asignando un mayor peso a este subgrupo.

Aunque la mayoría de reportes de estrategias de mantenimiento de cohortes derivan de países desarrollados 13,14,15,16,17,18,19,20,21, y rara vez son descritas experiencias donde los intentos de recuperación ocurren tras largos periodos sin contacto; algunas de las estrategias y los resultados obtenidos en nuestro protocolo de recuperación son similares a los reportados en el estudio desarrollado por Hurwitz et al. 17. Pese a que la población difiere ampliamente (136 niños y sus familias, incluidos en el año 2010 en Chicago, EE.UU.); los participantes de esta cohorte permanecieron unos 6 años sin contacto con los investigadores. En ese estudio se utilizaron secuencialmente: (1) llamadas telefónicas en diferentes horarios (hasta 2 intentos por cada número telefónico) y SMS, con tasa de contacto 55\%; (2) envío de correo electrónico o correspondencia a domicilio (contacto 19\%); (3) búsqueda en las redes sociales y bases de datos en línea -libres y pagadas- (contacto 16\%) 17. Allí la estrategia telefónica fue también el método que permitió la mayor tasa de recuperación, similar a esta experiencia. Sin embargo, nuestro protocolo requirió esfuerzos adicionales; tuvo un mayor número de intentos telefónicos $(5 v s$. 2 llamadas) por cada teléfono registrado, y contó con una escasa proporción de correos electrónicos disponibles para el contacto (2\%) en la línea de base. Asimismo, nuestro estudio contactó a 122 (vs. 68) participantes por mes, usando el doble de tiempo para su ejecución. Finalmente, mientras Hurwitz et al. 17 lograron una tasa de contacto global del 90\% con las 3 estrategias, nuestro estudio requirió de 2 PRP paralelos que incorporaron 4 estrategias para obtener una tasa de contacto del 76,5\%. 
Otros estudios norteamericanos reportan el uso de estrategias adicionales, como visitas domiciliarias, búsqueda en obituarios, documentos judiciales y registro de encarcelamiento, con secuencias de aplicación diferentes; que consistentemente reportan tasas de contacto superiores al 80\% 18,19,20,21. Las diferencias entre los métodos y resultados obtenidos en esos estudios y CHICAMOCHA podrían explicarse por el contexto geográfico, social y político y la temporalidad en que se desarrolla cada uno; con factores determinantes que favorecen el contacto en países desarrollados como: (1) la política de acceso abierto a información centralizada de contacto, que es incluso considerada como de interés público (v.g. teléfono y dirección); (2) mayor asignación presupuestaria a la investigación, que permite ejecutar los seguimientos con mayor consistencia, manteniendo intervalos de contacto regulares y más cortos con el participante; (3) la disposición y el acceso de las poblaciones a las herramientas tecnológicas, lo que permite una mejor calidad de los registros de la información de contacto.

Algunas experiencias de estudios en entornos de mayor similitud al nuestro son recopiladas en la revisión sistemática realizada por Hill 22, en la que se incluyeron 13 estudios longitudinales de Asia, África y Suramérica. La tasa promedio de contacto fue 74\% (rango 48\%-97\%), luego de 2-11 años 22, resultado similar a nuestro reporte. Aunque esta revisión no describe el uso de estrategias secuenciales de contacto en los estudios incluidos, se generan recomendaciones de algunos de los procedimientos individuales para localizar a los participantes, similares a los métodos empleados en este protocolo.

Además del resultado aditivo que mostró la aplicación de las estrategias secuenciales usadas en nuestro protocolo de recuperación de participantes, identificamos que las personas de mayor edad, estrato socioeconómico bajo y propietarios de vivienda fueron más propensos al re-contacto.

Estos resultados, son semejantes a los descritos en un estudio longitudinal desarrollado en 12.660 mujeres en Australia (con re-contacto del 76.5\% luego de 13 años). Allí las participantes más jóvenes, residentes urbanas, con menor nivel educativo y mayor estrés económico, tuvieron menor contacto en el seguimiento ${ }^{19}$. Otros estudios de cohorte, con diferentes poblaciones e intervalos de tiempo de contacto, reportan consistentemente que tener mayor edad es la característica que más frecuentemente está asociada al re-contacto 23,24,25,26.

Estos hallazgos podrían deberse principalmente a la disminución de la movilidad geográfica que se espera en grupos de mayor edad y propietarios de vivienda, puesto que se percibe un mayor estado de estabilidad. En contraste, las dificultades para aumentar la movilidad social en personas de bajo estrato socioeconómico en nuestro país, podrían explicar la baja movilidad geográfica en este grupo.

\section{Fortalezas y limitaciones}

La ejecución de este PRP enfrentó algunos desafíos; entre ellos, trabajar con presupuesto y personal limitado para el número de participantes a contactar, y contar con la logística necesaria para desarrollar con éxito las estrategias. Esto implicó apalancar recursos adicionales para entrenar y reunir la voluntad del personal, que realizó alrededor de 18.000 llamadas telefónicas (mediana de 9 llamadas por participante) y conformó cuadrillas de voluntarios que desarrollaron las visitas al domicilio y vecindario.

Por otro lado, nuestro estudio enfrentó desavenencias con la ley de protección de datos, que rige actualmente en nuestro país, cuya regulación impide el acceso a los datos personales de los usuarios de las EAPB, aún por motivos de investigación en salud. Este podría ser un método económico y de menor esfuerzo para conseguir información de participantes pertenecientes a estudios de cohorte.

Los resultados obtenidos en este estudio responden al contexto social e histórico en el cual se obtuvieron. Por ejemplo, no aplicaba para el momento la posibilidad de contacto mediante celular, correo electrónico o redes sociales, a los que los participantes hubieran dado acceso al estudio en línea de base. También estas tasas de recuperación son producto de una secuencia específica, basada en los recursos, los precedentes y la intuición del equipo del proyecto. Por tanto, pueden no ser extrapolables en un contexto, secuencia y temporalidad diferentes.

De los 1.258 participantes contactados después de los PRP, se obtuvieron nuevos números celulares en el $81,2 \%$ ( $n=1.021)$ y correo electrónico en el 48,3\% ( $\mathrm{n}=608)$, permitiendo que para futuros seguimientos esta información pueda ser utilizada para el contacto. 
En conclusión, las estrategias usadas dentro de los PRP permitieron la recuperación de aproximadamente el $76 \%$ de los participantes, dando viabilidad a la cohorte CHICAMOCHA, después de 12 años sin contacto. Consistentemente con los hallazgos descritos en la literatura, el contacto telefónico fue la estrategia de mayor rendimiento para lograr el re-contacto; lo que sugiere mantener esta estrategia como primera opción para el contacto en estudios longitudinales. Los individuos con características que aumentan la movilidad geográfica como una menor edad, no poseer vivienda propia o estrato socioeconómico medio-alto, podrían requerir de periodos de seguimiento más estrechos y estrategias de contacto adicionales a las propuestas en este PRP.

\section{Colaboradores}

S. M. Vásquez contribuyó sustancialmente a la concepción y diseño del trabajo, adquisición, análisis y interpretación de datos, a la redacción y revisión crítica del trabajo, a la aprobación de la versión final, y es responsable de todos los aspectos del trabajo que garantizan su calidad. V. M. Herrera y J. C. Villar contribuyeron sustancialmente a la concepción y diseño del trabajo, a la redacción y revisión crítica del trabajo, a la aprobación de la versión final, y son responsables de todos los aspectos del trabajo que garantizan su calidad.

\section{Agradecimientos}

Este estudio se desarrolló en el marco del proyecto Emergencia de Perfiles de Riesgo para Enfermedades no Comunicables en Adultos Colombianos. Incidencia y Factores de Riesgo para Hipertensión Arterial en la Cohorte CHICAMOCHA Luego de Diez Años de Seguimiento, financiado por COLCIENCIAS, contrato 489-2012. Agradecemos a los voluntarios que participaron en la ejecución de este protocolo: Belén Vega, Ximena Hernández, Laura Polo, Julián Ardila, Paula Alvarado, Yenny Castellanos, Nelson Pinto.

\section{Referencias}

1. Sedgwick P. Bias in observational study designs: prospective cohort studies. BMJ 2014; 349:g7731.

2. Hurtado AR. La financiación de la investigación en Colombia: desafíos y posibilidades. https://repository.javeriana.edu.co:8443/ bitstream/handle/10554/15179/Rios.pdf? sequence $=1 \&$ isAllowed $=y$ (accedido el 10/Feb/ 2018).

3. Instituto de Estadística, Organización de las Naciones Unidas para la Educación, la Ciencia y la Cultura; Banco Mundial. Gastos en investigación y desarrollo (\% del PIB). https://datos. bancomundial.org/indicador/GB.XPD.RSDV. GD.ZS (accedido el 10/Feb/2018).

4. Landriault E, Matlin SA; Global Forum for Health Research. Monitoring financial flows for health research 2009. Behind the global numbers. http://announcementsfiles.cohred. org/gfhr_pub/reports/2009_en.pdf (accedido el 11/Jul/2018).

5. World Health Organization. Public health, innovation and intellectual property: report of the Expert Working Group on Research and Development Financing. Executive board, 126th session, provisional agenda item 4.3. http://apps.who.int/gb/ebwha/pdf_files/ EB126/B126_6Add1-en.pdf?ua=1 (accedido el 13/Jul/2018).

6. Departamento Administrativo Nacional de Estadística. Colombia: estimación de la migración 1973-2005. http://biblioteca.dane.gov. co/media/libros/estudios_postcensales_06. PDF (accedido el 10/Feb/2018).

7. Congreso de Colombia. Ley Estatutaria 1581 de 2012. Disposiciones generales para la protección de datos personales. http://wsp.presidencia.gov.co/Normativa/Leyes/Documents/ LEY\%201581\%20DEL\%2017\%20DE\%20OC TUBRE\%20DE\%202012.pdf (accedido el 11/ $\mathrm{Jul} / 2018)$.

8. Villar JC. Challenges in identifying subclinical chronic chagasic cardiomyopathy in a Colombian Urban Population [Doctoral Dissertation]. Hamilton: McMaster University; 2006. 
9. Villar JC. Effects of a combined treatment with Itraconazole/Pravastatin and Allendronate for asymptomatic T. cruzi-infected individuals: a randomized controlled trial in the CHICAMOCHA Study. In: XVIII Congreso Internacional de Medicina Tropical y Malaria. Río de Janeiro: Instituto Oswaldo Cruz, Fundação Oswaldo Cruz; 2012. p. 561.

10. Villar JC. CHICAMOCHA 3 - equivalence of usual interventions for Trypanosomiasis (EQUITY) (CHICAMOCHA-3). Bucamaranga: Universidad Autónoma de Bucaramanga; 2015.

11. Villar JC, Herrera VM, Cháves AM, Martínez LX, Villar-Centeno LA, Ardila E, et al. Cardiovascular Health Investigation and Collaboration to Assess the Markers and Outcomes of Chagas disease (CHICAMOCHA): concepto, métodos y características de una cohorte de donantes de sangre en Bucaramanga, Colombia. MedUNAB 2015; 18:14-26.

12. American Association for Public Opinion Research. Standard definitions final dispositions of case codes and outcome rates for surveys. https://www.aapor.org/AAPOR_ Main/media/publications/Standard-Defini tions20169theditionfinal.pdf (accedido el 06/ $\mathrm{Jul} / 2018)$.

13. Robinson KA, Dennison CR, Wayman DM, Pronovost PJ, Needham DM. Systematic review identifies number of strategies important for retaining study participants. J Clin Epidemiol 2007; 60: 757-65.

14. Brueton VC, Tierney JF, Stenning S, Meredith S, Harding S, Nazareth I, et al. Strategies to improve retention in randomised trials: a Cochrane systematic review and meta-analysis. BMJ Open 2014; 4:e003821.

15. Booker CL, Harding S, Benzeval M. A systematic review of the effect of retention methods in population-based cohort studies. BMC Public Health 2011; 11:249.

16. Robinson KA, Dinglas VD, Sukrithan V, Yalamanchilli R, Mendez-Tellez PA, DennisonHimmelfarb C, et al. Updated systematic review identifies substantial number of retention strategies: Using more strategies retains more study participants. J Clin Epidemiol 2015; 68:1481-7.
17. Hurwitz LB, Schmitt KL, Olsen MK. Facilitating development research: suggestions for recruiting and re-recruiting children and families. Front Psychol 2017; 8:1525.

18. Cotter RB, Burke JD, Stouthamer-Loeber M, Loeber R. Contacting participants for followup: how much effort is required to retain participants in longitudinal studies? Eval Program Plann 2005; 28:15-21.

19. Lyons KS, Carter JH, Carter EH, Rush KN, Stewart BJ, Archbold PG. Locating and retaining research participants for follow-up studies. Res Nurs Health 2004; 27:63-8.

20. Dilworth-Anderson P, Williams SW. Recruitment and retention strategies for longitudinal African American caregiving research: the Family Caregiving Project. J Aging Health 2004; 16 (5 Suppl):137S-56S.

21. Abshire M, Dinglas VD, Cajita MI, Eakin MN, Needham DM, Himmelfarb CD. Participant retention practices in longitudinal clinical research studies with high retention rates. BMC Med Res Methodol 2017; 17:30.

22. Hill Z. Reducing attrition in panel studies in developing countries. Int J Epidemiol 2004; 33:493-8.

23. Powers J, Tavener M, Graves A, Loxton D. Loss to follow-up was used to estimate bias in a longitudinal study: a new approach. J Clin Epidemiol 2015; 68:870-6.

24. Littman AJ, Boyko EJ, Jacobson IG, Horton J, Gackstetter GD, Smith B, et al. Assessing nonresponse bias at follow-up in a large prospective cohort of relatively young and mobile military service members. BMC Med Res Methodol 2010; 10:99.

25. Ng SK, Scott R, Scuffham PA. Contactable non-responders show different characteristics compared to lost to follow-up participants: insights from an Australian Longitudinal Birth Cohort Study. Matern Child Health J 2016; 20:1472-84.

26. Koloski NA, Jones M, Eslick G, Talley NJ. Predictors of response rates to a long term followup mail out survey. PLoS One 2013; 8:e79179. 
Abstract

This article seeks to describe the results of a participant recovery protocol in the CHICAMOCHA cohort after 12 years of follow-up and to identify factors associated with re-contact. In a prospective cohort study with 1,644 participants in Bucaramanga, Colombia (mean age 36 years; standard deviation $=8.5$ years; $63 \%$ men), we implemented a participant recovery protocol that included 4 sequential strategies: (1) phone contact; (2) sending mail; (3) visiting the household; and (4) Publishing notices in the press local. Following steps 1-2, we attempted to update contact information through government databases in order to once again apply steps 1-2 for individuals who had not been contacted. We calculated crude and weighted rates of contact by participant recovery protocol strategy and overall return. We estimated the association between baseline participant characteristics and their state after participant recovery protocol through multiple logistical regression. We contacted 1,258 (76.4\%) participants; $65 \%(n=825)$ through phone contact. Weighted rates of contact were: $41 \%$ phone contact, $14.6 \%$ mail and $31 \%$ visits. Contact through newspaper ads was practically null. Age $>36$ years $(O R=1.48)$; low socioeconomic stratum $(O R=1.42)$ or being a home owner at baseline $(O R=2.05)$ were associated with recontact. Consistent with other descriptions, phone contact is the re-contacting strategy with greatest returns in longitudinal studies. Individuals with characteristics that increase geographical mobility may require shorter follow-up periods and additional contact strategies.

Follow-Up Studies; Patient Selection; Patient Compliance; Refusal to Treat

\section{Resumo}

Este artigo trata de descrever os resultados de um protocolo de recuperação dos participantes na coorte CHICAMOCHA, após 12 anos sem acompanhamento, e identificar fatores associados ao re-contato. Em um estudo de coorte prospectiva com 1.644 participantes em Bucaramanga, Colômbia (idade media 36 anos; desvio padrão $=8.5$ anos; 63\% homens), foi implementado protocolo de recuperação dos participantes que incluiu quatro estratégias sequenciais: (1) contato telefônico; (2) envio de correspondência; (3) visita ao domicílio; $e$ (4) publicação de avisos na imprensa. Depois dos passos 1-2, tentou-se refrescar a informação de contato em bases de dados governamentais para voltar a aplicar paralelamente a sequência 1-2 nas pessoas que não foram contatadas. Foram calculadas as taxas de contacto brutas e ponderadas por estratégia do protocolo de recuperação dos participantes e o rendimento global. Foi estimada a associação entre as características dos participantes na linha de base e o seu estado depois do protocolo de recuperação dos participantes por meio de regressão logística múltipla. Foram contatados 1.258 $(76,4 \%)$ participantes; o $65 \%(n=825)$ através de contacto telefônico. As taxas de contato ponderadas foram: $41 \%$ contato telefônico, 14,6\% correspondência e $31 \%$ visitas. O contato através de avisos no jornal foi virtualmente nulo. Uma idade > 36 anos $(O R=1,48)$; estrato socioeconômico baixo $(O R=1,42)$ ou ser proprietário de casa na linha de base $(O R=2,05)$ foram associados ao re-contato. Consistente com outras descrições, o contato telefônico é a estratégia do re-contato com maior rendimento em estudos longitudinais. Os indivíduos com características que aumentam a mobilidade geográfica poderiam precisar de períodos de acompanhamento mais estreitos e estratégias de contacto adicionais.

Seguimentos; Seleção de Pacientes; Cooperação do Paciente; Recusa do Médico a Tratar
Recibido el 23/Mar/2018

Versión final presentada el 30/Jul/2018

Aprobado el 21/Sep/2018 